\title{
The Infection Rate of COVID-19 in Wuhan, China: Combined Analysis of Population Samples
}

Hui-Qi Qu ${ }^{1}, \mathrm{MD}, \mathrm{PhD}$; Zhangkai Jason Cheng ${ }^{1,2}, \mathrm{PhD}$; Zhifeng Duan ${ }^{1,3}, \mathrm{PhD}$; Lifeng Tian ${ }^{1}$, PhD; Hakon Hakonarson ${ }^{1,4}$, $\mathrm{MD}, \mathrm{PhD}$ \author{
States \\ Corresponding Author: \\ Hakon Hakonarson, MD, PhD \\ Center for Applied Genomics \\ The Children's Hospital of Philadelphia \\ 3615 Civic Center Boulevard \\ Philadelphia, PA \\ United States \\ Phone: 12674260088 \\ Email: Hakonarson@email.chop.edu
}

${ }^{1}$ Center for Applied Genomics, The Children's Hospital of Philadelphia, Philadelphia, PA, United States

${ }^{2}$ Department of Radiation Oncology, Sun Yat-sen University Cancer Center, Guangzhou, China

${ }^{3}$ Institute of Allergy and Immunology, School of Medicine, Shenzhen University, Shenzhen, China

${ }^{4}$ Divisions of Human Genetics and Pulmonary Medicine, The Perelman School of Medicine, University of Pennsylvania, Philadelphia, PA, United

\section{Abstract}

Background: The coronavirus disease (COVID-19) pandemic began in Wuhan, China, in December 2019. Wuhan had a much higher mortality rate than the rest of China. However, a large number of asymptomatic infections in Wuhan may have never been diagnosed, contributing to an overestimated mortality rate.

Objective: This study aims to obtain an accurate estimate of infections in Wuhan using internet data.

Methods: In this study, we performed a combined analysis of the infection rate among evacuated foreign citizens to estimate the infection rate in Wuhan in late January and early February.

Results: Based on our analysis, the combined infection rate of the foreign evacuees was 0.013 (95\% CI 0.008-0.022). Therefore, we estimate the number of infected people in Wuhan to be 143,000 (range 88,000-242,000), which is significantly higher than previous estimates. Our study indicates that a large number of infections in Wuhan were not diagnosed, which has resulted in an overestimated case fatality rate.

Conclusions: Increased awareness of the original infection rate of Wuhan is critical for proper public health measures at all levels, as well as to eliminate panic caused by overestimated mortality rates that may bias health policy actions by the authorities.

(J Med Internet Res 2020;22(8):e20914) doi: 10.2196/20914

\section{KEYWORDS}

infectious disease; COVID-19; infection rate; China; Wuhan; fatality; public health; diagnosis

\section{Introduction}

In December 2019, the first cases of coronavirus disease (COVID-19) were reported in Wuhan, China, a megacity with a population of approximately 11 million people. To prevent the spread of this highly infectious disease, the government initiated a city-wide lockdown on January 23, 2020. However, despite these efforts, COVID-19 spread to many countries across the world, reaching pandemic levels, and continues to be a serious public health concern due to its high mortality rate.
According to the large-sample analysis by $\mathrm{Wu}$ and McGoogan [1], China's case fatality rate (CFR) was $2.3 \%$ - that is, 1023 deaths from 44,672 confirmed cases as of February 11, 2020, with a significant proportion of cases originating from Wuhan. The large number of infected people in Wuhan put a tight strain on essential medical resources. The city had a much higher mortality rate (according to Feb 10th statistics: $\mathrm{CFR}=4.05 \%$ [748 deaths/18,454 diagnoses]; Apr 24th statistics: $\mathrm{CFR}=7.69 \%$ [3869 deaths/50,333 diagnoses]) than the rest of China. The overall CFR of $2.3 \%$ for China was likely overestimated, due 
to strained medical resources and a large number of undiagnosed patients. According to a recent study, $78 \%$ of those who had been infected were asymptomatic [2]. Therefore, a large number of asymptomatic infections in Wuhan might have never been diagnosed, which contributed to the overestimated CFR. An accurate estimation of the infection rate is therefore important to assess Wuhan's CFR precisely.

\section{Methods}

Using Markov Chain Monte Carlo methods, Wu et al [3] estimated that 75,815 individuals $(95 \%$ CI 37,304-130,330) had been infected in Wuhan as of January 25, 2020. Following this, a number of foreign governments evacuated their citizens and performed thorough etiological tests on them. This group of evacuees can serve as a "random" sample to estimate the infection rate in Wuhan. With internet search as an important source of epidemiologic information on COVID-19 [4], we performed a combined analysis of the infection rates of these population samples using publicly available data (Table 1), instead of a simple pooled calculation, considering potential differences in lifestyles and pathogen exposure across different populations. The combined analysis was done using the Comprehensive Meta-Analysis Software (Biostat, Inc).

Table 1. Number of infected people from different countries.

\begin{tabular}{llll}
\hline Country & Evacuation date & Confirmed cases (n=14), & Evacuees $(\mathrm{n}=1401), \mathrm{n}$ \\
\hline Japan [5] & N/A & 9 & 566 \\
Korea [6-8] & January 31, 2020 & 1 & 368 \\
Germany [9] & February 1, 2020 & 2 & 124 \\
Singapore [10-12] & January 30, 2020 & 1 & 92 \\
Italy [13] & February 2, 2020 & 1 & 56 \\
United States [14] & January 29, 2020 & 0 & 195 \\
\hline
\end{tabular}

${ }^{\mathrm{a} N} / \mathrm{A}$ : not applicable.

\section{Results}

Our analysis demonstrates that there is no significant heterogeneity across different population samples (heterogeneity

Figure 1. Combined analysis of infection rates of different populations. test $P=.491)$. The combined infection rate is $0.013(95 \% \mathrm{CI}$ 0.008-0.022) (Figure 1). Based on our results, we estimate the number of infected people in Wuhan, China, to be 143,000 (range 88,000-242,000), which is significantly higher than the estimate proposed by $\mathrm{Wu}$ et al [3].
Study name

$\begin{array}{lccccrr} & \begin{array}{c}\text { Event } \\ \text { rate }\end{array} & \begin{array}{c}\text { Lower } \\ \text { limit }\end{array} & \begin{array}{c}\text { Upper } \\ \text { limit }\end{array} & \text { Z value } & \boldsymbol{P} \text { value } \\ \text { Japan } & 0.016 & 0.008 & 0.030 & -12.277 & .000 \\ \text { Korea } & 0.003 & 0.000 & 0.019 & -5.897 & .000 \\ \text { Germany } & 0.016 & 0.004 & 0.062 & -5.767 & .000 \\ \text { Singapore } & 0.011 & 0.002 & 0.073 & -4.486 & .000 \\ \text { Italy } & 0.018 & 0.003 & 0.116 & -3.971 & .000 \\ \text { United States } & 0.003 & 0.000 & 0.039 & -4.215 & .000 \\ & 0.013 & 0.008 & 0.022 & -16.371 & .000\end{array}$

\section{Event rate and $95 \% \mathrm{Cl}$}

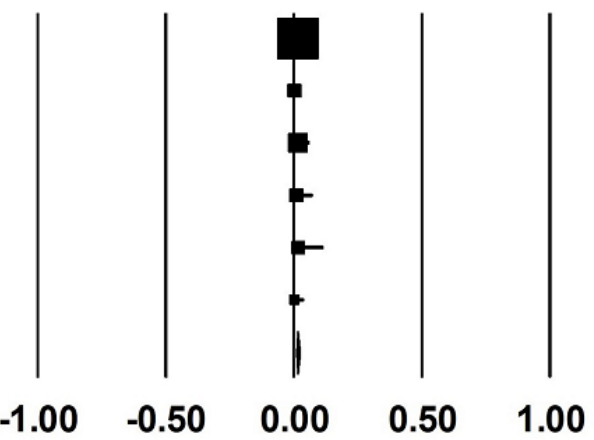

Favors A Favors B

\section{Discussion}

Our estimate indicates that a large number of infections in Wuhan were not diagnosed. The number of undiagnosed cases in late January and early February is larger than the final diagnosed count reported to date $(n=50,333)$, which has resulted in an overestimated CFR. In addition, our study suggests that the lower CFR $(0.51 \%)$ estimated by the Centre for Evidence-Based Medicine [15] does not indicate viral variants and loss of virulence. Taken together, increased awareness of 
the original infection rates in Wuhan, China, is critically important for appropriate public health measures at all levels, as well as to eliminate panic caused by overestimated mortality rates that may bias health policy actions by the authorities.

\section{Acknowledgments}

The study was supported by Institutional Development Funds from the Children's Hospital of Philadelphia to the Center for Applied Genomics, The Children's Hospital of Philadelphia Endowed Chair in Genomic Research to HH.

\section{Authors' Contributions}

HQ was responsible for the literature search, study design, data collection, data analysis, data interpretation, and writing; ZJC was involved in data collection; ZD in data interpretation; LT in study design and data interpretation; and HH in study design, data interpretation, and writing.

\section{Conflicts of Interest}

None declared.

\section{References}

1. Wu Z, McGoogan JM. Characteristics of and Important Lessons From the Coronavirus Disease 2019 (COVID-19) Outbreak in China: Summary of a Report of 72314 Cases From the Chinese Center for Disease Control and Prevention. JAMA 2020 Feb 24:e. [doi: 10.1001/jama.2020.2648] [Medline: 32091533]

2. Day M. Covid-19: four fifths of cases are asymptomatic, China figures indicate. BMJ 2020 Apr 02;369:m1375. [doi: 10.1136/bmj.m1375] [Medline: 32241884 ]

3. Wu JT, Leung K, Leung GM. Nowcasting and forecasting the potential domestic and international spread of the 2019-nCoV outbreak originating in Wuhan, China: a modelling study. The Lancet 2020 Feb 29;395(10225):689-697. [doi:

10.1016/S0140-6736(20)30260-9] [Medline: 32014114]

4. Higgins TS, Wu AW, Sharma D, Illing EA, Rubel K, Ting JY, Snot Force Alliance. Correlations of Online Search Engine Trends With Coronavirus Disease (COVID-19) Incidence: Infodemiology Study. JMIR Public Health Surveill 2020 May 21;6(2):e19702 [FREE Full text] [doi: 10.2196/19702] [Medline: 32401211]

5. Ministry of Health, Labor and Welfare. About the present situation of new type coronavirus infectious disease and correspondence of Ministry of Health, Labor and Welfare (February 7, 2019 version). 2019. URL: https://www.mhlw.go.jp/ stf/newpage 09396.html [accessed 2020-02-12]

6. KBS World Radio. The second batch of Wuhan evacuation test results were negative, the first batch of 1 person diagnosed with infection. 2020. URL: http://world.kbs.co.kr/service/news view.htm?lang=c\&Seq Code=66621 [accessed 2020-02-12]

7. Minister of Health and Welfare. New Coronavirus Infectious Disease Central Accident Headquarters Routine New Release. 2020. URL: https://www.mohw.go.kr/eng/nw/nw0101vw.

jsp?PAR MENU ID=1007\&MENU ID=100701\&page=1\&CONT SEQ=352718 [accessed 2020-02-12]

8. Ministry of Health and Welfare. Domestic occurrence of new coronavirus (February 2). 2020. URL: http://www.mohw.go.kr/ react/al/sal0301vw.jsp?PAR MENU ID=04\&MENU ID=0403\&page=3\&CONT SEQ=352645 [accessed 2020-02-12]

9. DW. Coronavirus: German evacuation flight from China carried two infected people. 2020. URL: https://www.dw.com/ en/coronavirus-german-evacuation-flight-from-china-carried-two-infected-people/a-52229955 [accessed 2020-02-12]

10. APF. Coronavirus: countries evacuate citizens from China. New Straits Times. 2020. URL: https://www.nst.com.my/world/ world/2020/01/561086/coronavirus-countries-evacuate-citizens-china [accessed 2020-02-12]

11. Wei TT. Some Singaporeans with symptoms of virus staying behind in Wuhan even as 92 are evacuated. Straits Times. 2020. URL: https://www.straitstimes.com/singapore/ some-singaporeans-with-symptoms-of-virus-staying-behind-in-wuhan-even-as-92-are-evacuated [accessed 2020-02-12]

12. Yong C. Coronavirus: 2 new cases in S'pore, including Certis officer who had served quarantine orders on 2 who tested positive. Straits Times. 2020. URL: https://www.straitstimes.com/singapore/ coronavirus-2-new-cases-in-singapore-including-certis-cisco-staff-who-had-served [accessed 2020-02-12]

13. Seckin B. Italy reports third confirmed case of coronavirus. Anadolu Agency. 2020. URL: https://www.aa.com.tr/en/europe/ italy-reports-third-confirmed-case-of-coronavirus/1726934 [accessed 2020-02-12]

14. Waldrop T, Yan H. The 1st group of Americans evacuated from Wuhan and quarantined over coronavirus just got released. CNN. 2020. URL: https://edition.cnn.com/2020/02/10/us/coronavirus-american-evacuees-release/index.html [accessed 2020-02-12]

15. Oke J, Heneghan C. Global Covid-19 Case Fatality Rates. Centre for Evidence-Based Medicine. 2020. URL: https://www. cebm.net/covid-19/global-covid-19-case-fatality-rates/ [accessed 2020-06-12]

\section{Abbreviations}

COVID-19: coronavirus disease 
CFR: case fatality rate

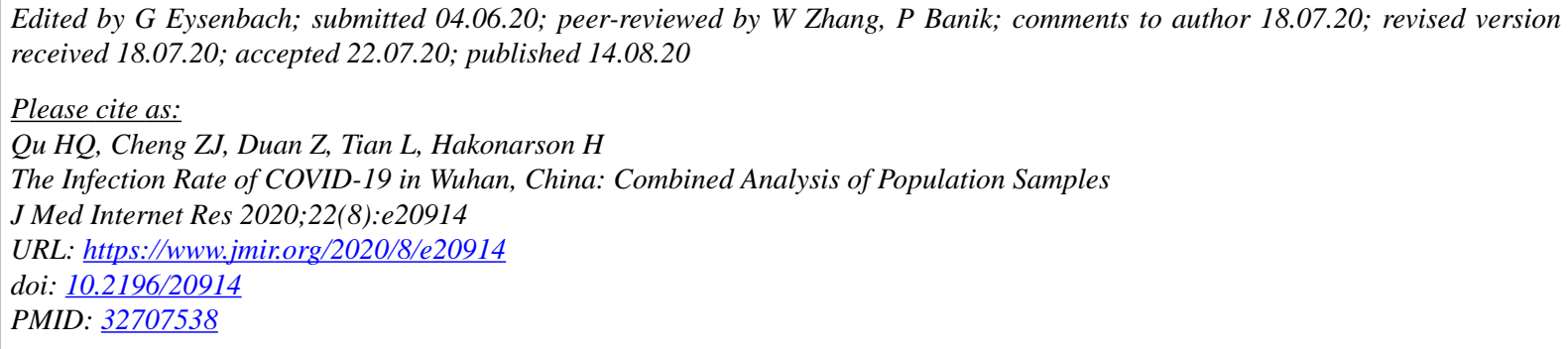

CHui-Qi Qu, Zhangkai Jason Cheng, Zhifeng Duan, Lifeng Tian, Hakon Hakonarson. Originally published in the Journal of Medical Internet Research (http://www.jmir.org), 14.08.2020. This is an open-access article distributed under the terms of the Creative Commons Attribution License (https://creativecommons.org/licenses/by/4.0/), which permits unrestricted use, distribution, and reproduction in any medium, provided the original work, first published in the Journal of Medical Internet Research, is properly cited. The complete bibliographic information, a link to the original publication on http://www.jmir.org/, as well as this copyright and license information must be included. 